\title{
Genetic algorithm based optimization of overcurrent relay coordination for improved protection of DFIG operated wind farms
}

\begin{abstract}
Rigorous protection of wind power plants is an immensely momentous aspect in electrical power protection engineering which must be contemplated thoroughly during designing the wind plants to afford a proper protection for power components in case of fault occurrence. The most commodious protection apparatus are overcurrent relays (OCRs) which are responsible for protecting power systems from impending faults. These relays are set and coordinated with each other by applying IEEE or IEC standards methods, however, their operation times are relatively long and the coordination between these relays are critical. The other common problem in wind farm protection systems is when a fault occurs in a plant, several OCRs operate instead of a designated relay to that particular fault location. This undesirable action can result in unnecessary power loss and disconnection of healthy feeders out of the plant which is extremely dire. Therefore, this research proposes a novel genetic algorithm (GA) based optimization for proper coordination of OCRs to improve their functions for protection of wind farms. GA optimization technique has some advantages over other intelligent algorithms including high accuracy, fast response and most importantly achieving optimal solutions for nonlinear characteristics of OCRs. In this work the improvement in protection of wind farm is achieved through optimizing the relay settings, reducing their operation time, time setting multiplier of each relay, improving the coordination between relays after implementation of IEC 60255-151:2009 standard. It is found that the new approach achieves significant improvement in operation of OCRs at the wind farm and drastically reduces the accumulative operation time of the relays.
\end{abstract}

Keyword: Wind farm; Power system protection; Overcurrent relay; Optimization of relay coordination; Genetic algorithm 